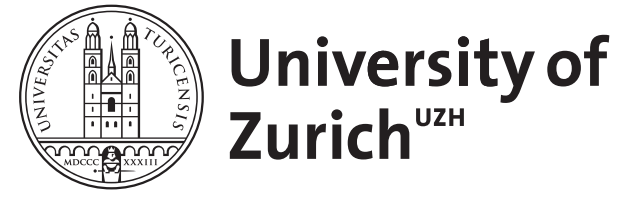

Zurich Open Repository and Archive

University of Zurich

Main Library

Strickhofstrasse 39

CH-8057 Zurich

www.zora.uzh.ch

Year: 2012

\title{
Polycythaemia vera
}

Gemünden, Cornelia ; Gerber, Bernhard

DOI: https://doi.org/10.1024/1661-8157/a000928

Posted at the Zurich Open Repository and Archive, University of Zurich

ZORA URL: https://doi.org/10.5167/uzh-71675

Journal Article

Accepted Version

Originally published at:

Gemünden, Cornelia; Gerber, Bernhard (2012). Polycythaemia vera. Praxis, 101:631-641.

DOI: https://doi.org/10.1024/1661-8157/a000928 
DOI 10.1024/1661-8157/a000928

((PX10_Rubrik CME))

\section{Polycythaemia vera}

\section{Im Artikel verwendete Abkürzungen}

$\begin{array}{ll}\text { AML } & \text { Akute myeloische Leukämie } \\ \text { EPO } & \text { Erythropoietin } \\ \text { ET } & \text { Essentielle Thrombozythämie } \\ \text { MPN } & \text { MyeloproliferativeNeoplasien } \\ \text { MF } & \text { Primäre Myelofibrose } \\ \text { PV } & \text { Polyzythaemia vera }\end{array}$

Die Polycythaemiavera ist durch eine Erythrozytose beruhend auf einem klonalen Stammzelldefekt definiert. Dies kann im klinischen Bild zu Plethora, Pruritus, Erythromelalgien, Mikrozirkulationsstörungen, Splenomegalie und thrombembolischen Ereignissen führen.

Die Polycythaemia vera (PV) gehört zu der Gruppe der myeloproliferativen Neoplasien (MPN). Sie beruht auf einem klonalen Stammzelldefekt der Hämatopoiese, bei der die Erythropoietin (EPO)-unabhängige Proliferation der Erythropoiese mit daraus resultierender Zunahme der Erythrozytenmasse im Vordergrund steht. Daneben findet sich häufig eine Neutrophilie und Thrombozytose als Zeichen einer gesteigerten Granulo- und Megakaryopoiese. Zu Beginn der Erkrankung zeigt sich nicht selten wegen der gleichzeitigen PlasmavolumenExpansion keine relevante Erythrozytose und nur eine nuklearmedizinisch fassbare Erhöhung der Erythrozytenmasse. Bei Erstdiagnose steht dann aber die Erythrozytose im Vordergrund. Nach Jahren bis Jahrzehnten, kommt es im fortgeschrittenen Stadium (panzytopenische Spätphase) oft zur Splenomegalie, Zeichen extramedullärer Blutbildung, Knochenmarkfibrose und Zytopenien. Das typische Erkrankungsalter bei Erstdiagnose liegt zwischen dem 60. und 65. Lebensjahr, Männer erkranken etwas häufiger als Frauen. Die Lebenserwartung ist unter Therapie gegenüber der Normalbevölkerung nur leicht vermindert (15-Jahres Überleben 65\%). Ohne Therapie lag in einer Arbeit aus den frühen 60er Jahren das mediane Überleben nach Erstdiagnose mit 6-18 Monaten hingegen deutlich tiefer, wobei diese Angaben aus heutiger Sicht etwas pessimistisch scheinen. Das Risiko für einen Übergang in eine post-polyzythämische Myelofibrose beträgt 25-35\% nach 20 Jahren und das Risiko für einen Übergang in eine akute myeloische Leukämie (AML) je nach Therapie und Literaturangaben 5-15\% nach zehn Jahren.

Die Pathogenese der PV ist noch nicht vollständig geklärt. Im Jahre 2005 wurde bei Patienten mit PV eine Punktmutation im Exon 14 (V617F) des JAK2-Gens identifiziert. Diese Mutation führt zu einer Aktivitätszunahme der Tyrosinkinase JAK2 und damit unter anderem zur EPO-unabhängigen Proliferation der Erythropoiese. Die JAK2 V617F-Mutation ist bei $>95 \%$ der Patienten mit PV nachweisbar, weitere sehr seltene Mutationen konnten bereits identifiziert werden (v.a. im Exon 12). Das Auftreten von Mutationen im JAK2-Gen scheint aber nicht das eigentlich auslösende Ereignis in der Pathogenese der PV darzustellen. Zudem ist die JAK2 V617F-Mutation nicht spezifisch für die PV sondern auch bei anderen MPN nachweisbar (ca. je 50\% der MF und ET).

Nichtsdestotrotz ist der Nachweis der JAK2 V617FMutation heute ein zentrales Diagnosekriterium der PV (,major' Kriterium gemäss WHO 2008). 


\section{Q}

\section{Klinik ((Ü2))}

Bedingt durch die verschiedenen hämatologischen Veränderungen (erhöhter Hämatokrit, quantitativ und qualitativ veränderte Thrombozyten, Neutrophilie) kann es zu neurologischen Symptomen wie Tinnitus, Schwindel, Kopfschmerzen, Sehstörungen und weiteren Mikrozirkulationsstörungen sowie thrombembolischen Ereignissen kommen. Mikrozirkulationsstörungen führen zu Erythromelalgien, Livedo reticularis oder digitalen Ischämien (Abb. 1). Bei Erstdiagnose beschreiben ca. 25\% der Patienten einen aquagenen Pruritus. Im Untersuchungsstatus findet sich gelegentlich eine Splenomegalie und Plethora. Seltener und v.a. bei extremer Thrombozytose besteht eine Blutungsneigung. Eine sekundäre Gicht und arterielle Hypertonie sind weitere mögliche Begleitsymptome der PV.

Im fortgeschrittenen Stadium klagen die Patienten vor allem über konstitutionelle Symptome und abdominelle Beschwerden wie Völlegefühl und Inappetenz, meist bedingt durch die zunehmende Splenomegalie.

Abb. 1: Chronischer Grosszehenarterienverschluss bei PV mit distaler trockener Nekrose.

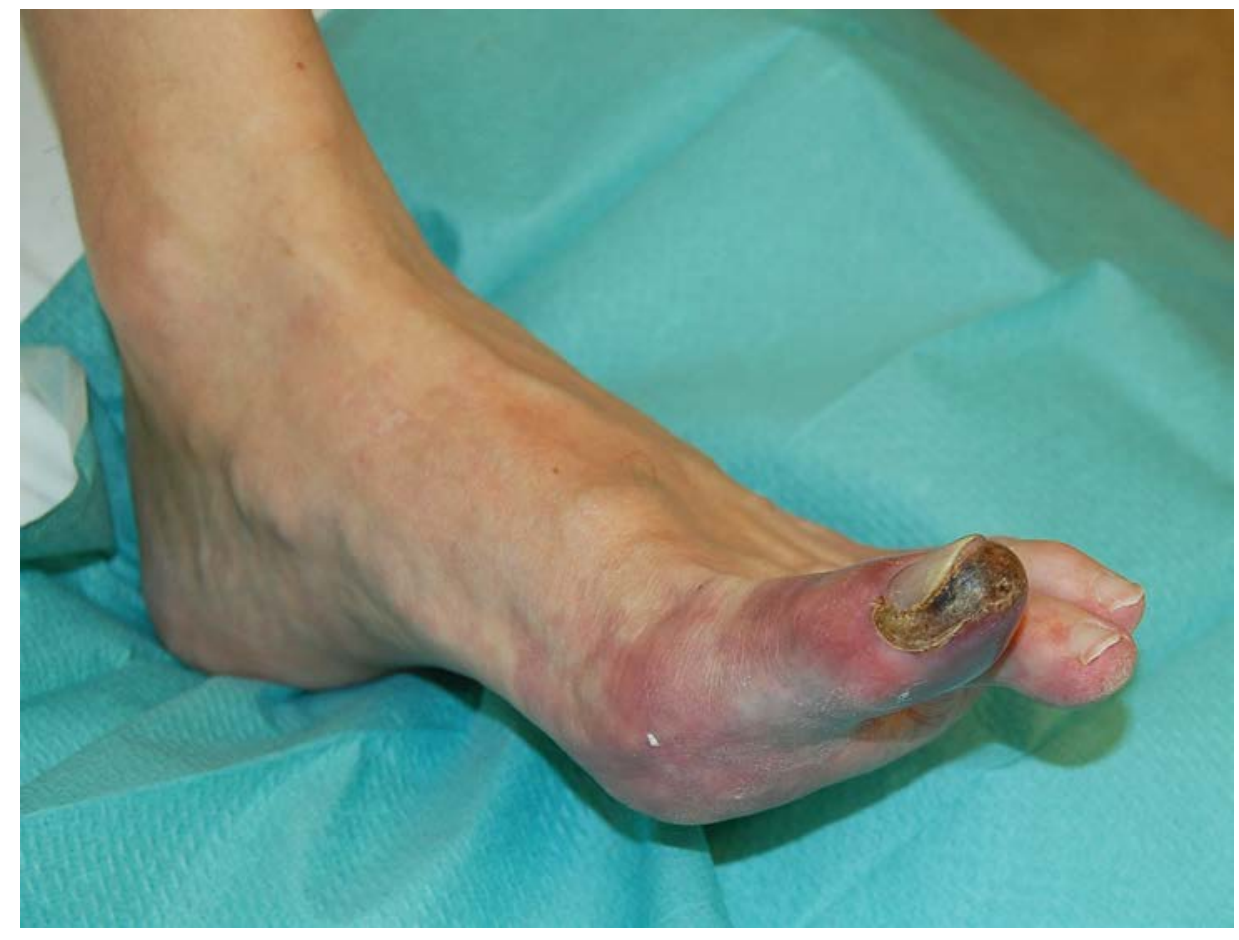

\section{Komplikationen ((Ü2))}

Das Auftreten arterieller oder venöser Thrombembolien kann Initalsymptom sein und ist zugleich die häufigste Komplikation bei PV-Patienten. Thrombotische Komplikationen wie Myokardinfarkte, zerebrovaskuläre Insulte oder venöse Thrombembolien stellen die häufigsten Todesursachen dar. Viszeralvenenthrombosen sind gefürchtete und typische Komplikationen von Patienten mit MPN. Die Rate thrombembolischer Ereignisse liegt mit 3-5\% pro Jahr bei Diagnosestellung und 20-40\% im Gesamtverlauf über demjenigen der Normalbevölkerung. Das Thrombembolierisiko steigt erwartungsgemäss mit zunehmendem Lebensalter und ist für Patienten mit stattgehabter Thrombembolie und deutlicher Neutrophilie erhöht. Die Rate an Blutungen liegt unter 5\%, die Häufigkeit schwerer Blutungen noch tiefer. Das Auftreten von Blutungen wird v.a. bei hoher Thrombozytenzahl 
beobachtet und ist durch eine Thrombozytenfunktionstörung und/oder einen erworbenen von Willebrand-FaktorMangel begünstigt. Wie eingangs erwähnt, beträgt das Risiko für einen Übergang in eine post-polyzythämische Myelofibrose 25-35\% nach 20 Jahren und das Risiko für einen Übergang in eine AML je nach Therapie und Literaturangaben 5-15\% nach zehn Jahren. 
Abklärungsstrategie ((Ü1))

Ausgangspunkt der Abklärung ist die erhöhte Hämoglobinkonzentration (bei Frauen $\mathrm{Hb}>165 \mathrm{~g} / \mathrm{l}$, bei Männern $>185 \mathrm{~g} / \mathrm{l}$ ) resp. die nukleramedizinisch bestimmte, erhöhte Erythrozytenmasse (>125\% der Norm für beide Geschlechter), sei es als Zufallsbefund oder bei klinischem Verdacht. Der wichtigste Schritt in der Diagnosestellung ist die Abgrenzung der PV gegenüber sekundären Erythrozytosen. Hier sind neben der gezielten Anamnese und der Berücksichtigung des Hb-Verlaufes die Bestimmung der ErythropoietinKonzentration im Serum und die Suche nach einer JAK2 V617F Mutation aus dem peripheren Blut richtungsweisend (Abb. 2) [1].

Die Anamnese soll die häufigsten bei der PV vorkommenden Symptome wie Kopfschmerzen, Schwindel, Juckreiz, Mikrozirkulationsstörungen, arterielle und venöse Thombembolien beinhalten und Anhaltspunkte auf evtl. sekundäre Ursachen liefern (Rauchgewohnheiten, Hinweise auf Herz- oder Lungenkrankheiten, maligne Tumoren, Medikamente). Die minimale körperliche Untersuchung schliesst die Herz-Lungenuntersuchung, die Untersuchung der Milzgrösse und der Leber und die Suche nach Mikrozirkulationsstörungen mit ein. Unserer Empfehlungen zur initialen Labor- und apparativen Diagnostik bei Verdacht auf PV sind in Tabelle 1 festgehalten:

Abb. 2: Abklärungsstrategie bei Erythrozytose basierend auf JAK2 V617F-Mutationsstatus und Erythropoietinkonzentration (adaptiert nach [1]).

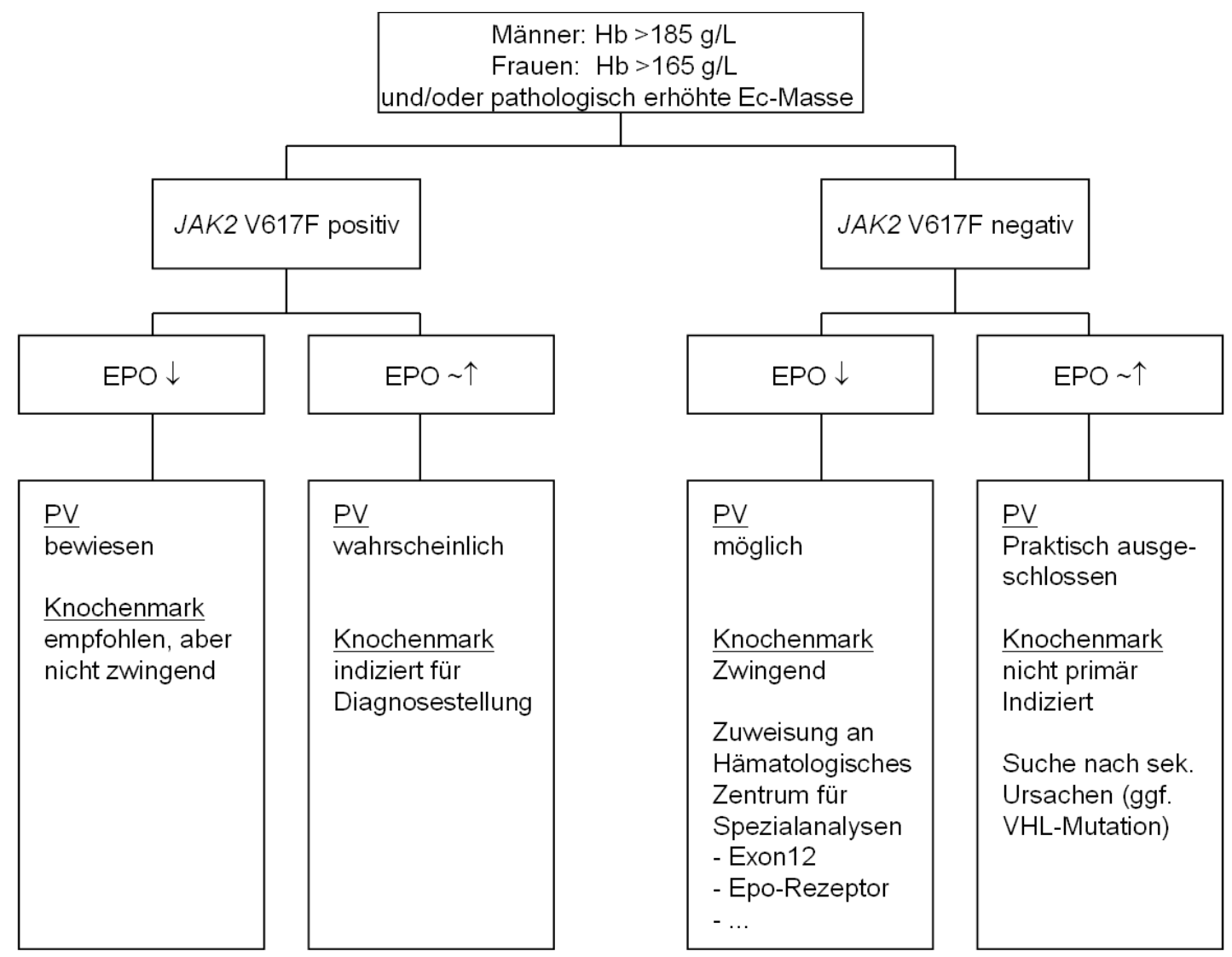

((Layout: oberstes Kästli beide Einheiten g/l anstatt g/L)) 


\begin{tabular}{|l|}
\hline Tab. 1: Basisdiagnostik bei vermuteter PV \\
\hline Manuelles Differenzialblutbild und Retikulozyten \\
\hline Ferritin, Vitamin B12, Folsäure im Erythrozyten \\
\hline Leberwerte (AST, ALT, alkalische Phosphatase, LDH, Bilirubin) \\
\hline Nierenfunktion (Kreatinin) und Harnsäure \\
\hline INR, aPTT, Fibrinogen (Cave: Verfälschung der Werte bei Hämatokrit-Werten>60\%) \\
\hline Erythropoietin-Konzentration im Serum \\
\hline JAK2 V617F-Mutation aus dem peripheren Blut \\
\hline Pulsoxymetrische Sauerstoffsättigung ggf. arterielle Blutgasanalyse (inkl. COHb und p50) \\
\hline Abdomen-Sonographie \\
\hline Thorax-Röntgenbild \\
\hline EKG \\
\hline
\end{tabular}

Typischerweise findet sich im peripheren Blut eine unauffällige Erythrozytenmorphologie, gelegentlich besteht aber schon bei Diagnosestellung aufgrund des hohen Verbrauches ein Eisenmangel mit Hypochromasie und Mikrozytose der Erythrozyten. Eine allfällige Leukozytose ist meist durch eine Neutrophilie bedingt, eine relevante Ausschwemmung von myeloischen Vorstufen und Erythroblasten (leukerythroblastäres Blutbild) wird bei Übergang zu einer Myelofibrose beobachtet. Die Thrombozyten sind typischerweise anisogranulär und anisozytär mit immer wieder grossen Formen (Riesenthrombozyten).

Eine Knochenmarkpunktion ist für die Diagnose einer PV nicht immer notwendig (vgl. Diagnosekriterien), wird aber bei uns am Universitätsspital Zürich zur Diagnosesicherung und zur Beurteilung einer eventuell bestehenden Knochenmarkfibrose vor Therapiebeginn grundsätzlich durchgeführt. Die Knochenmarkuntersuchung ist als Ausgangswert für die Verlaufsbeurteilung hilfreich, insbesondere bei der Frage nach einem Übergang in eine post-PV Myelofibrose. Klassischerweise zeigt sich ein proliferatives, hyperzelluläres Knochenmark mit Steigerung aller drei Zellreihen, prädominanter Erythropoiese und clusterartig angeordneten form- und grössenvariablen Megakaryozyten (Abb. 3). Bei ca. 20\% der Patienten mit PV finden sich zusätzliche zytogenetische Aberrationen. Der Stellenwert der Zytogenetik ist aber im Vergleich zu anderen hämatologischen Neoplasien noch gering. Das Anlegen von endogenen erythroiden Kolonien ist zur Diagnosestellung meist nicht mehr zwingend notwendig, ist aber in den aktuellen WHO-Diagnosekriterien weiterhin enthalten.

Weisen die klinischen und im Labor erhobenen Befunde eher in Richtung einer sekundären Erythrozytose, so muss die entsprechende Grunderkrankung internistisch weiter abgeklärt werden. Gelegentlich ist die Abgrenzung der PV von sekundären Erythrozytosen oder anderen hämatologischen Krankheiten, die mit einer Erythrozytose einhergehen schwierig. Hier empfehlen wir die Zusammenarbeit mit einem hämatologischen Zentrum. Spezialanalysen wie z.B. Bestimmung der Sauerstoffaffinitätskurve, Mutationen im EPO-Rezeptor oder im von Hippel-Lindau-Gen (z.B. Chuvash-Anomalie) sowie die Suche nach Exon12-Mutationen im JAK2-Gen können in Einzelfällen indiziert sein. Gelegentlich lohnt sich auch eine Bestimmung des BCR-ABL1-Fusionstranskriptes, wenn die Abgrenzung gegenüber anderen MPN schwierig ist. Nicht immer kann bei Diagnosestellung eine eindeutige Entität bestimmt werden: Es gibt Übergangsformen zwischen ET und PV und auch das hyperproliferative präfibrotische Stadium einer Myelofibrose kann initial PV-ähnlich imponieren. In diesen Fällen gibt jeweils der Verlauf Aufschluss über die eigentliche Krankheitsentität.

Abb. 3: Typische Knochenmarkbiopsie bei PV (Methakrylat-Schnitt, HE-Färbung) mit Steigerung der Granulopoiese, Thrombopoiese und Erythropoiese sowie gruppierten teilweise atypischen Megakaryozyten. 


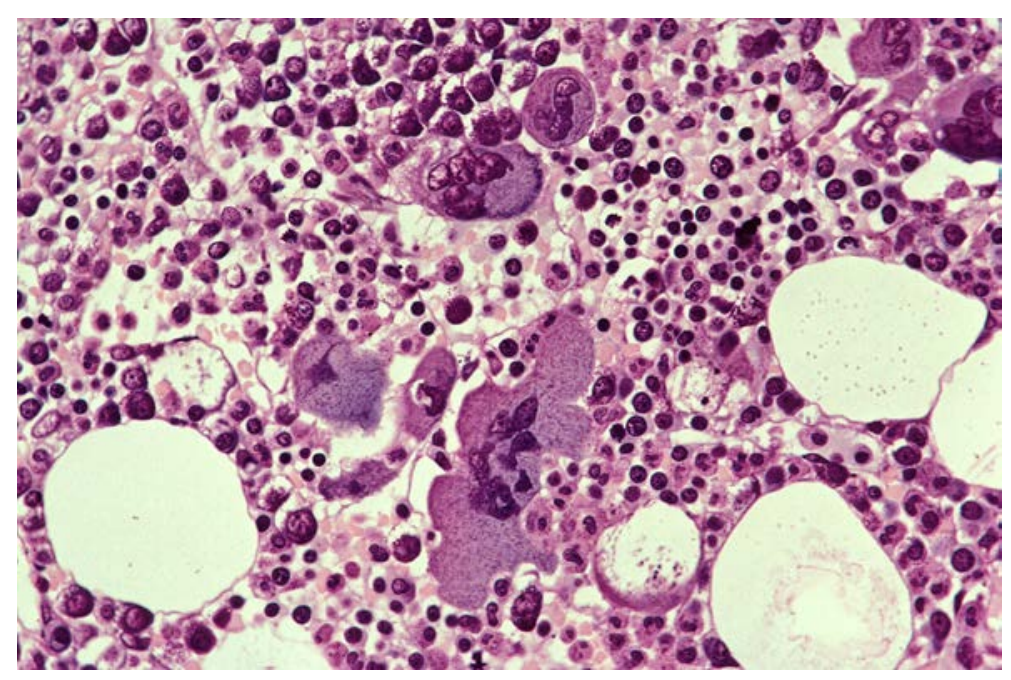


Diagnosekriterien/Ursachen/Differenzialdiagnose ((Ü1))

\section{Diagnosekriterien ((Ü2))}

Die Diagnose einer PV wird aktuell unter Anwendung der WHO 2008 Kriterien gestellt (Tab. 2) [1

\begin{tabular}{|l|l|}
\hline Tab. 2: WHO 2008 Kriterien für PV Diagnostik \\
\hline Hauptkriterien & $\begin{array}{l}\text { 1. Hämoglobin>165 g/l (Frauen), >185 g/l (Männer) oder eine nuklearmedizinisch } \\
\text { gemessene pathologisch erhöhte Erythrozytenmasse }\end{array}$ \\
\cline { 2 - 2 } & $\begin{array}{l}\text { 2. Nachweis der JAK2 V617F-Mutation oder einer anderen funktionell ähnlichen Mutation } \\
\text { (z.B. JAK2 Exon12) }\end{array}$ \\
\hline Nebenkriterien & $\begin{array}{l}\text { 1. Hyperzelluläres Knochenmark mit Steigerung von Erythropoiese, Granulopoiese und } \\
\text { Megakaryopoiese }\end{array}$ \\
\cline { 2 - 2 } & 2. Supprimiertes Erythropoietin \\
\cline { 2 - 2 } & 3. Nachweis von endogenen erythroiden Kolonien in vitro \\
\hline
\end{tabular}

Die Diagnose PV darf gestellt werden wenn (i) beide Hauptkriterien und ein Nebenkriterium oder (ii) das erste Hauptkriterium und 2 Nebenkriterien vorliegen.

\section{Differenzialdiagnose ((Ü2))}

Wie bereits erwähnt, muss die PV differenzialdiagnostisch von den insgesamt häufiger auftretenden sekundären Erythrozytosen (früher Polyglobulien) abgegrenzt werden. Hier liegt meist eine internistische Grunderkrankung vor: Bei chronischen Herz- und Lungenerkrankungen kommt es durch die arterielle Hypoxie zu einer sekundären Erythrozytose (z.B. Schlafapnoe-Syndrom). Bei Leber- und Nierenerkrankungen (inkl. Hämangiomen, Tumoren, etc.) kann die Erythrozytose durch eine vermehrte EPO-Produktion entstehen. Erythrozytosen können aber auch bei Exsikkose auftreten, hierbei handelt es sich um sog. Pseudoerythrozytosen, bei denen tatsächlich eine Verminderung des Plasmavolumens vorliegt. Zu den Pseudoerythrozytosen zählt auch das Gaisböck-Syndrom resp. die «Stress-Erythrozytose». Diese Zustände können mittels nuklearmedizinischer Untersuchungen (Doppelvolumenbestimmung) diagnostiziert werden.

Daneben gibt es seltene kongenitale Erythrozytosen, die auf einer Veränderung des Sauerstoff-Sensings der PHD-HIF-VHL-Achse beruhen (z.B. die Chuvash-Mutation des VHL-Gens).Desweiteren können EPO-Rezeptor Mutationen, ein 2,3-DPG-Mangel und Hämoglobinvarianten mit abnormer Sauerstoffaffinität (z.B. Hämoglobin Zürich Albisrieden) zu einer angeborenen Erythrozytose führen. 
†herapie/Prognose/Prävention [2,3] ((Ü1))

\section{Therapie ((Ü2))}

Ein kurativer Therapieansatz ist nur durch eine allogene Stammzelltransplantation möglich, was aufgrund des normalerweise recht günstigen Krankheitsverlaufes und der mit der Stammzelltransplantation assoziierten Morbidität und Mortalität nur bei einem kleinen Teil der Patienten v.a. mit Übergang in eine post-PV-Myelofibrose oder sekundäre AML indiziert ist. Das primäre Ziel der Therapie ist bei der Mehrzahl der Betroffenen die Symptomlinderung und vor allem die Reduktion des Risikos für thrombembolische Ereignisse. Basis der Behandlung sind Aderlässe und die Gabe von Acetylsalicylsäure (ASS). Man beginnt mit Aderlässen von ca. $400 \mathrm{ml}$ in dreitägigen bis wöchentlichen Abständen, bis der Hämatokrit unter 45\% gesenkt ist. Wir verwenden diese Hämatokrit-Grenze unabhängig vom Geschlecht des Patienten. Obwohl meist als Grenzwert genutzt, ist diese Grenze nie in klinischen Studien prospektiv validiert worden. Als Folge regelmässiger Aderlässe kommt es zu einem gewollten Eisenmangel (dieser wird nicht substituiert). Zur weiteren Therapieoptimierung werden die Patienten in Risikokategorien für thrombembolische Ereignisse eingeteilt (Tab. 3).

\begin{tabular}{|l|l|}
\hline \multicolumn{2}{|l|}{ Tab. 3: Risiko für Thrombembolien bei PV } \\
\hline Tiefes Risiko & $\begin{array}{l}\text { Alter <60 Jahre } \\
\text { Keine Thrombembolien in Vorgeschichte } \\
\text { Keine kardiovaskulären Risikofaktoren }\end{array}$ \\
\hline Intermediäres Risiko & $\begin{array}{l}\text { Alter <60 Jahre } \\
\text { Keine Thrombembolien in der Vorgeschichte } \\
\text { Kardiovaskuläre Risikofaktoren (Diabetes mellitus, arterielle Hypertonie, Rauchen, } \\
\text { Dyslipidämie, positive Familienanamnese) }\end{array}$ \\
\hline Hohes Risiko & $\begin{array}{l}\text { Alter }>60 \text { Jahre und/oder } \\
\text { Stattgehabte Thrombembolien }\end{array}$ \\
\hline
\end{tabular}

Patienten >60 Jahre und Patienten mit stattgehabter Thrombembolie haben ungeachtet der anderen Risikofaktoren ein erhöhtes Risiko für Thrombembolien, weshalb hier neben der Aderlasstherapie und der Gabe von Acetylsalicylsäure eine zytoreduktive Therapie erfolgen sollte (Abb. 4). Auch für Patienten mit kardiovaskulären Risikofaktoren kann eine zytoreduktive Therapie erwogen werden.

Die Neutrophilie und Thrombozytose stellen bei der PV an und für sich keine harten Kriterien für den Beginn einer zytoreduktiven Therapie dar. Meist wird aber eine Zytoreduktion bei der PV ab einer Leukozytenzahl von >25 g/l und einer Thrombozytenzahl von >1000 g/l empfohlen. Eine erhöhte Neutrophilenzahl ist mit einem höheren Thromboserisiko vergesellschaftet und eine hohe Thrombozytenzahl durch veränderte ThrombozytenEigenschaften und einen möglichen sekundären von Willebrand-Faktor-Mangel mit einem erhöhten Blutungsrisiko. Bei jüngeren Patienten wird dies im klinischen Alltag aber unterschiedlich gehandhabt, auch wegen des leukämogenen Potenzials der zytoreduktiven Behandlung.

Hydroxyurea (Litalir®) typischerweise in einer Dosierung von $1000 \mathrm{mg} / \mathrm{Tag}$ ist noch immer die initiale zytoreduktive Therapie der Wahl für die Mehrzahl der Patienten. Die häufigsten Nebenwirkungen sind Zytopenien sowie seltener Ulzerationen der Haut und der Schleimhäute. Bei jüngeren Patienten (<40Jahre), Schwangeren, Patienten mit ausgeprägtem Pruritus und bei Unverträglichkeit von Hydroxyurea kann Interferon alpha (z.B. Roferon $\AA-A$ ) in einer initialen Dosis von 3x 3Mio E pro Woche eingesetzt werden [4]. Allerdings ist dieses Medikament für die Indikation PV in der Schweiz nicht kassenpflichtig und es muss vorgängig eine Kostengutsprache beim Versicherer eingeholt werden. Insbesondere bzgl. des Auftretens von Zytopenien, 
Knochenmarkfibrose und zur Therapie des Pruritus scheint Interferon alpha den anderen Therapieoptionen überlegen zu sein. Ausserdem erhofft man sich natürlich ein deutlich geringeres leukämogenes Risiko. Um die Therapieadhärenz für Interferon zu erhöhten, braucht es eine gute Information der Patienten über die zu erwartenden «grippeartigen» unerwünschten Wirkungen (Müdigkeit, Fieber, Schüttelfrost, Appetitlosigkeit, Muskelschmerzen, Kopfweh, Gelenkschmerzen, Schwitzen). Diese Symptome können mit entsprechender Prämedikation (Paracetamol, nicht-steroidale Antirheumatika) gemildert werden und nehmen in ihrem Schweregrad in der Regel mit der Zeit ab. Als wichtige mögliche unerwünschte Wirkung ist das Auslösen von affektiven Störungen (Depression) zu erwähnen. Rund ein Drittel der Patienten toleriert die Behandlung nicht. Anagrelid (z.B. Thromboreductin ${ }^{\circledR}$ oder Xagrid®), das bei der ET zugelassen ist und nur die Thrombozytenzahl senkt, wird bei schwer kontrollierbaren Thrombozytosen meist in Kombination mit weiteren zytoreduktiven Medikamenten und Aderlasstherapie verwendet. Eine weitere Therapiealternative ist Pipobroman (Vercyte®), das in der Schweiz nicht ohne weiteres erhältlich ist und speziell importiert werden muss. In einer kürzlich publizierten Studie mit >16 Jahren medianem Follow-up wurde für Pipobroman im vgl. zu Hydroxyurea aber ein signifikant höheres Leukämierisiko gefunden [5]. Die Radiophosphor-Therapie ist wirksam und kann nach wie vor in Einzelfällen v.a. für ältere Patienten (>70 bis 80 Jahre) als Therapiealternative evaluiert werden, ist aber heutzutage sicher nicht die Behandlung der ersten Wahl, da sie ebenfalls mit einem erhöhten Leukämierisiko vergesellschaftet ist. Neuere Medikamente wie z.B. die viel diskutierten JAK2-Inhibitoren sind zur Zeit nur im Rahmen von klinischen Studien erhältlich und daher nach wie vor einem sehr selektionierten Patientengut vorbehalten.

Abb. 4: Empfehlung zur initialen Therapie bei PV basierend auf der Einschätzung des Thrombembolie-Risikos, des Patientenalters, der klinischen Präsentation (Pruritus), der Medikamentenverträglichkeit und evtl.

Schwangerschaft. Wichtig: Interferon alpha ist für die Indikation PV in der Schweiz zur Zeit nicht kassenpflichtig, es muss also vor Therapiebeginn eine Kostengutsprache bei der Krankenkasse eingeholt werden.

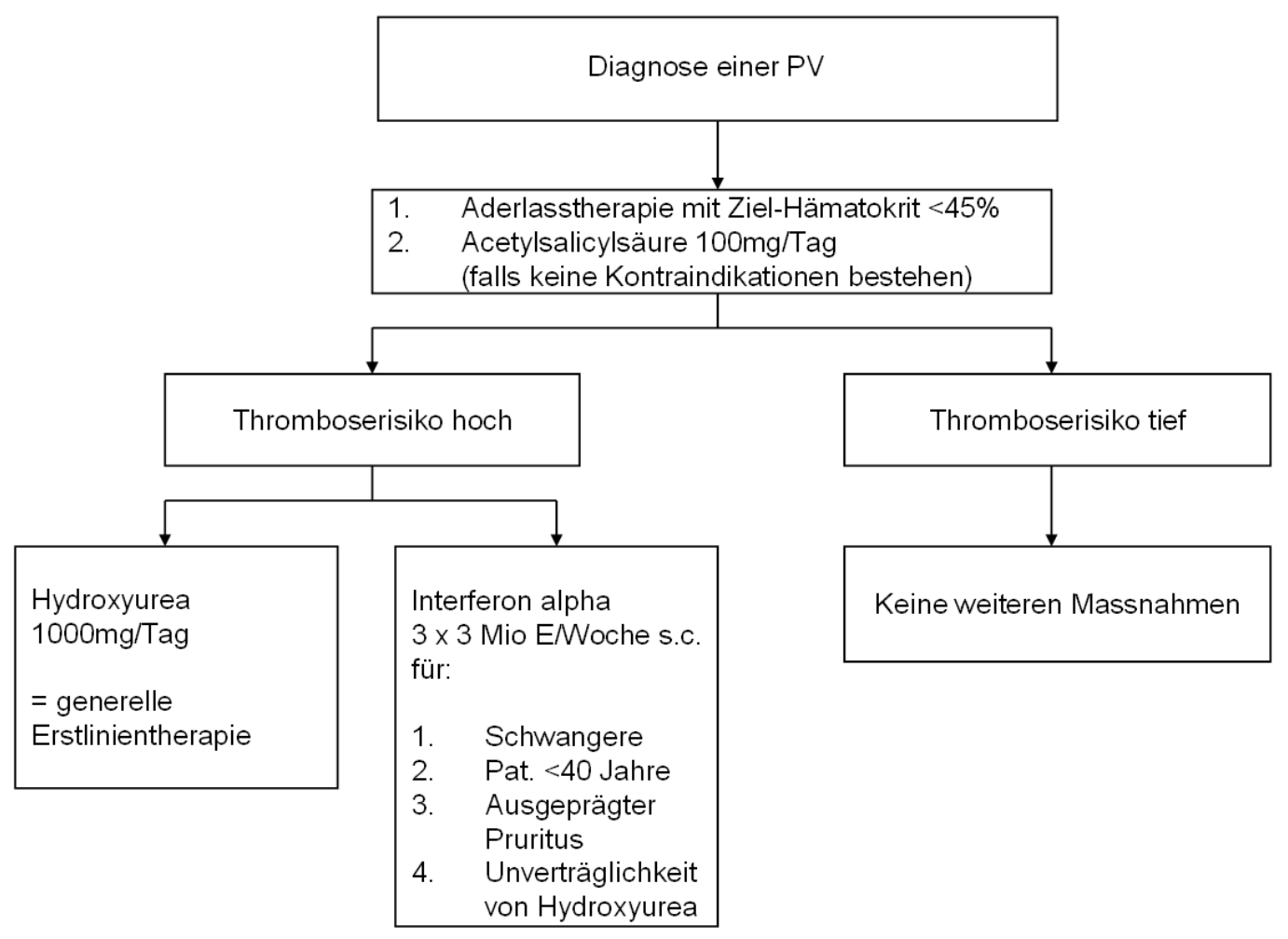


((Layout: 2. Kästli: $100 \mathrm{mg} / \mathrm{Tag}$; Kästli unten rechts: $1000 \mathrm{mg} / \mathrm{d}$; Kästli unten Mitte: $3 \times 3$ Mio E/Woche)) Verlaufskontrollen ((Ü2))Bei Therapieeinleitung sollten wöchentliche Blutbild-Kontrollen, resp. Aderlässe erfolgen. Bei stabiler Krankheit ohne Bedarf für Aderlässe können die Intervalle auf 1-3 Monate ausgedehnt werden. Klinische Verlaufskontrollen mit Differenzialblutbild und klinischer Kontrolle der Milzgrösse sollten alle sechs Monate erfolgen. Bei Befund-Änderung, B-Symtpomen, deutlicher Zunahme der Milzgrösse, neuen Zytopenien oder Zytosen sollte eine Knochenmarkpunktion zur weiteren Diagnostik durchgeführt werden. 


\section{Fallbericht zur Polycythaemia vera}

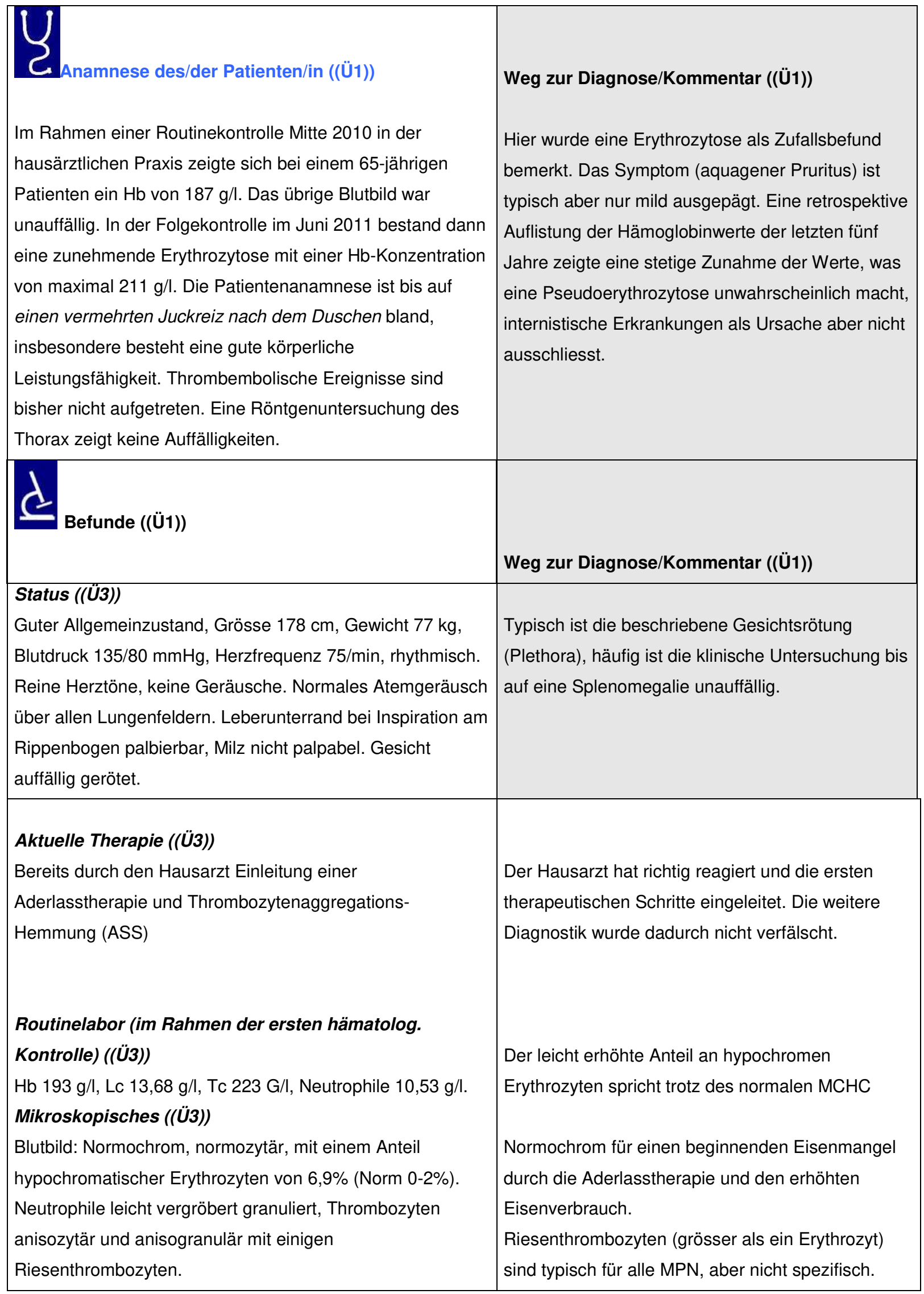


$\mathrm{Na} 140 \mathrm{mmol} / \mathrm{l}, \mathrm{K} 3,5 \mathrm{mmol} / \mathrm{l}$, Calcium 2,38 mmol/l, Krea $94 \mu \mathrm{mol} / \mathrm{l}, \mathrm{CRP} 11 \mathrm{mg} / \mathrm{l}, \uparrow$ Ferritin $25 \mathrm{~g} / \mathrm{l}$.

\section{Erweitertes Labor ((Ü3))}

JAK2 V617F-Mutation: positiv

Erythropoietin: 2 U/I (Norm 3-15)

\section{Weitere Befunde ((Ü3))}

Knochenmark-Aspirat: Deutlich hyperzellulär mit gesteigerter, linksverschobener Erythro- und Myelopoiese mit guter Durchreifung. Die Megakaryopoiese ist ebenfalls gesteigert mit in Bröckelnähe gruppierten Formen und milden morphologischen Atypien. In der Knochenmarkbiopsie keine Fibrose. In einer Berliner-BlauFärbung verminderte Eisenspeicher

Knochenmark-Zytogenetik: 46, XY

Milzgrösse: $12 \times 7 \times 4,5 \mathrm{~cm}$
Auffällig ist der nur knapp normale Ferritinwert bei leicht erhöhtem CRP, was für reduzierte Eisenspeicher spricht. In diesem Kontext im Rahmen der Aderlasstherapie und des erhöhten Verbrauches.

Gemäss WHO -Kriterien 2008 steht mit der Erfüllung der beiden Hauptkriterien $(\mathrm{Hb}$ Konzentration >185 g/l und JAK2 V617F-Mutation positiv) sowie einem minor-Kriterium (Erythropoietin supprimiert) die Diagnose einer PV fest. Auf die Anlage erythroider Kolonien wurde verzichtet.

Typischer Befund einer PV ist die trilineäre Steigerung der Hämatopoiese. Die Verminderung der Eisenspeicher ist bestätigt.

Nur 20\% der Patienten zeigen zusätzliche zytogenetische Veränderungen.

Die Milz ist minim vergrössert.

Aufgrund des fortgeschrittenen Lebensalters (>60 Jahre) besteht ein erhöhtes Risiko für TE, so dass eine zusätzliche Therapie mit Hydroxyurea eingeleitet wurde. Zudem wurde die Aderlasstherapie mit Zielhämatokrit $<45 \%$ sowie ASS beibehalten. 


\section{Fragen zur Polycythaemia vera}

\section{Frage 1}

\section{Welche Aussage zur PV ist nicht zutreffend?}

(Einfachauswahl, 1 richtige Antwort)

a) Die PV gehört in die Gruppe der myeloproliferativen Neoplasien.

b) Meist tritt die Erkrankung nach dem 60. Lebensjahr auf.

c) Typischerweise lässt sich das BCR-ABL1-Fusionstranskript nachweisen.

d) Es handelt sich um eine klonale Stammzellerkrankung.

e) Ein Hauptkriterium ist die Eryhrozytose.

\section{Frage 2}

Welches Diagnosekriterium muss gemäss WHO-Kriterien 2008 immer vorliegen, damit die Diagnose PV überhaupt gestellt werden darf?

(Einfachauswahl, 1 richtige Antwort)

a) Hämoglobin >185 $\mathrm{g} / \mathrm{l}$ bei Männern, $>165 \mathrm{~g} / \mathrm{l}$ bei Frauen oder eine nuklearmedizinsch nachgewiesene gesteigerte Erythrozytenmasse

b) Nachweis einer JAK2 V617F-Mutation

c) Hyperzelluläres Knochenmark mit Steigerung der Gesamthämatopoiese

d) Supprimierter Erythopoietin-Spiegel

e) Nachweis von endogenen erythroiden Kolonien in vitro

\section{Frage 3}

Welcher diagnostische Schritt gehört nicht zur Basisuntersuchung bei unklarer Erythrozytose?

(Einfachauswahl, 1 richtige Antwort)

a) Differenzialblutbild

b) Röntgenthorax

c) Klinischer Status einschliesslich Dokumentation der Milzgrösse

d) Knochenmarkpunktion

e) Bestimmung der JAK2 V617F-Mutation

\section{Frage 4}

Ein 70jähriger Mann stellt sich bei seinem Hausarzt zur Routinekontrolle vor. Die persönliche Anamnese bezüglich thrombembolischer Ereignisse ist bland. Der klinische Untersuchungsbefund ist bis auf eine auffallend rosige Gesichtsfarbe und eine Splenomegalie von $4 \mathrm{~cm}$ unter dem Rippenbogen unauffällig. Im peripheren Blutbild zeigt sich ein erhöhter Hämatokrit von 59\%, eine Hb-Konzentration von $188 \mathrm{~g} / \mathrm{l}$, Thrombozyten von $250 \mathrm{~g} / \mathrm{l}$ und Leukozyten von $14 \mathrm{~g} / \mathrm{l}$ (davon 85\% neutrophile Granulozyten) ohne Ausschwemmung myeloischer Vorstufen.

Sollte sich in den weiteren Abklärungen der Verdacht einer Polycythaemia vera bestätigen, was wäre eine adäquate Primärtherapie?

(Einfachauswahl, 1 richtige Antwort)

a) Aderlasstherapie

b) Thrombozytenaggregationshemmer 
c) Aderlasstherapie plus Thrombozytenaggregationshemmer

d) Aderlasstherapie plus Thrombozytenagregationshemmer plus Hydroxyurea

e) Aderlasstherapie plus Thrombozytenaggregationshemmer plus Anagrelide

\section{Frage 5}

Sie betreuen die 60-jährige Frau X. seit fünf Jahren aufgrund einer PV mit zwei monatlichen durchgeführten Aderlässen sowie Therapie mit Acetylsalicylsäure (ASS) in Ihrer Praxis. Darunter konnte bisher ein stabiler Hämatokrit von 45\% erreicht werden. Anlässlich der heutigen Konsultation berichtet Ihnen die Patientin über zunehmende Müdigkeit, Gewichtsverlust und Abgeschlagenheit, zudem habe sie seit zwei Wochen einen produktiven Husten. Im Blutbild zeigt sich ein $\mathrm{Hb}$ von $8,7 \mathrm{~g} / \mathrm{l}$ bei einem Hämatokrit von $38 \%$, die Thrombozyten sind bei $92 \mathrm{~g} / \mathrm{l}$, die Leukozyten bei 29,32. Im Blutausstrich zeigt sich eine Linksverschiebung der neutrophilen Granulozyten mit Ausschwemmung von Vorstufen und blastären Elementen. Die Thrombozyten sind anisozytär, die Erythrozytenmorphologie ist mikro- bis normozytär.

\section{Was veranlassen Sie als nächstes?}

(Einfachauswahl, 1 richtige Antwort)

a) Bestimmung des Ferritins und anschliessende Eisensubstitution

b) Einleitung einer zytoreduktiven Therapie mit Hydroxyurea

c) Knochenmarkpunktion einschliesslich zytogenetischer Untersuchungen

d) Einschluss des Patienten in eine klinische Studie mit JAK2-Inhibitoren

e) Erneute Kontrolle in zwei Wochen nach Abklingen des vermuteten pulmonalen Infektes 


\section{Korrespondenzadresse}

Dr. med. Bernhard Gerber

Klinik für Hämatologie

Universitätsspital Zürich

Rämistrasse 100

8091 Zürich

Bernhard.Gerber@usz.ch.

\section{Autoren}

Klinik für Hämatologie, Universitätsspital Zürich

Dr. med. Cornelia Gemünden, Dr. med. Bernhard Gerber

\section{Dank}

Wir danken Dr. med. Jeroen Goede, Oberarzt Hämatologie, Universitätsspital Zürich für die kritische Durchsicht des Manuskripts, die wertvollen fachlichen Inputs und das Überlassen der Abbildung der Knochenmarkbiopsie.

\section{Bibliographie}

1. Tefferi A, Vardiman JW: Classification and diagnosis of myeloproliferative neoplasms: the 2008 World Health Organization criteria and point-of-care diagnostic algorithms. Leukemia 2008; 22: 14-22.

2. Finazzi G, Barbui T: How I treat patients with polycythemia vera. Blood 2007; 109: 5104-5111.

3. Finazzi G, Barbui T: Evidence and expertise in the management of polycythemia vera and essential thrombocythemia. Leukemia 2008; 22: 1494-1502.

4. Kiladjian JJ, Mesa RA, Hoffman R: The renaissance of interferon therapy for the treatment of myeloid malignancies. Blood 2011; 117: 4706-4715.

5. Kiladjian JJ, Chevret S, Dosquet C, Chomienne C, Rain JD: Treatment of polycythemia vera with hydroxyurea and pipobroman: final results of a randomized trial initiated in 1980. J Clin Oncol 2011; 29: 39073913 
DOI 10.1024/1661-8157/a000929

((PX11_Rubrik CME-Antworten))

\section{Antworten zu den Fragen zur Polycythaemia vera aus PRAXIS Nr.}

\section{0}

\section{Frage 1}

Antwort c) ist richtig.

Das BCR-ABL1-Fusionstranskriptist beweisend für das Vorliegen einer CML und wird bei den anderen myeloproliferativen Neoplasien nicht nachgewiesen. Alle anderen Aussagen zur PV sind zutreffend.

\section{Frage 2}

\section{Antwort a) ist richtig.}

Die Erythrozytose ist der zentrale Punkt in den Diagnosekriterien der WHO von 2008. Alle anderen Kriterien sind wichtig, jedoch darf ohne Vorliegen einer Erythrozytose die Diagnose nicht gestellt werden.

\section{Frage 3}

Antwort d) ist richtig.

Obwohl für die Diagnose einer Polycythaemia vera als «minor»-Kriterium von zentraler Bedeutung, muss die Knochenmarkpunktion nicht zwingend bei Erstkontakt mit dem Patienten, also in den sogenannten Basisuntersuchungen durchgeführt werden. Wir am Universitätsspital Zürich führen bei den Patienten mit PV grundsätzlich vor Therapiebeginn eine Knochenmarkuntersuchung durch. Auch ist die Knochenmarkanalyse bei unklaren Erythrozytosen ohne sicheren anderen Auslöser eine unabdingbare Untersuchung.

\section{Frage 4}

\section{Antwort d) ist richtig.}

Sofern sich bei diesem Patienten die Diagnose einer PV bestätigt, muss der Patient bedingt durch das fortgeschrittene Patientenalter in die Gruppe der High-Risk-Patienten, d.h. Patienten mit hohem Risiko für thrombembolische Ereignisse, zugeordnet werden. Auch wenn bisher keine Thrombembolien aufgetreten sind und in den Angaben keine Hinweise für eine Myelofibrose bestehen. Die Antworten a) und c) sind für Patienten mit niedrigem TE-Risiko geeignet, Antwort b) ist als Basistherapie nicht ausreichend. Der Einsatz von Anagrelid sollte bei der PV nicht als Primärtherapie gewählt werden.

\section{Frage 5}

Antwort c) ist richtig.

Bei dieser Patientin sollte eine Knochenmarkpunktion erfolgen, um eine akute myeloische Leukämie auszuschliessen. Der beschriebene Leistungsknick und die Veränderungen im peripheren Blutbild sind suggestiv für eine akute myeloische Leukämie. Es sollte daher auch nicht das Abklingen des vermuteten pulmonalen Infektes abgewartet werden, da akute myeloische Leukämien rasch progredient verlaufen können und eine bereits aufgetretene Infektkomplikation ohne adäquate Behandlung der Grundkrankheit schwerlich in den Griff zu kriegen sein wird.

Eine Progression der Erkrankung zu einer post-PV-Myelofibrose ist ebenfalls denkbar und die Diagnose könnte ebenfalls mittels Knochenmarkpunktion gestellt werden.

Ein Eisenmangel bei Patienten mit Aderlasstherapie ist Teil des Therapiekonzeptes und sollte nur bei Feststellung einer Anämie und auch dort nur vorsichtig durch Substiution korrigiert werden. Eine Substitution bei 
normaler Hämoglobinkonzentration führt bei PV zu einem raschen Anstieg der Hämoglobinkonzentration und würde somit den Effekt der Aderlasstherapie zunichte machen. Gelegentlich ist bei Patienten mit ausgeprägter Thombozytose und Anämie unter Hydroxyurea die Gabe von i.v. Eisen teil des Therapiekonzeptes. 